Document downloaded from:

http://hdl.handle.net/10251/38101

This paper must be cited as:

Gregori Gregori, V.; Morillas, S.; Roig, B. (2013). Fuzzy quasi-metrics for the Sorgenfrey line. Fuzzy Sets and Systems. 222:98-107. doi:10.1016/j.fss.2012.11.001.

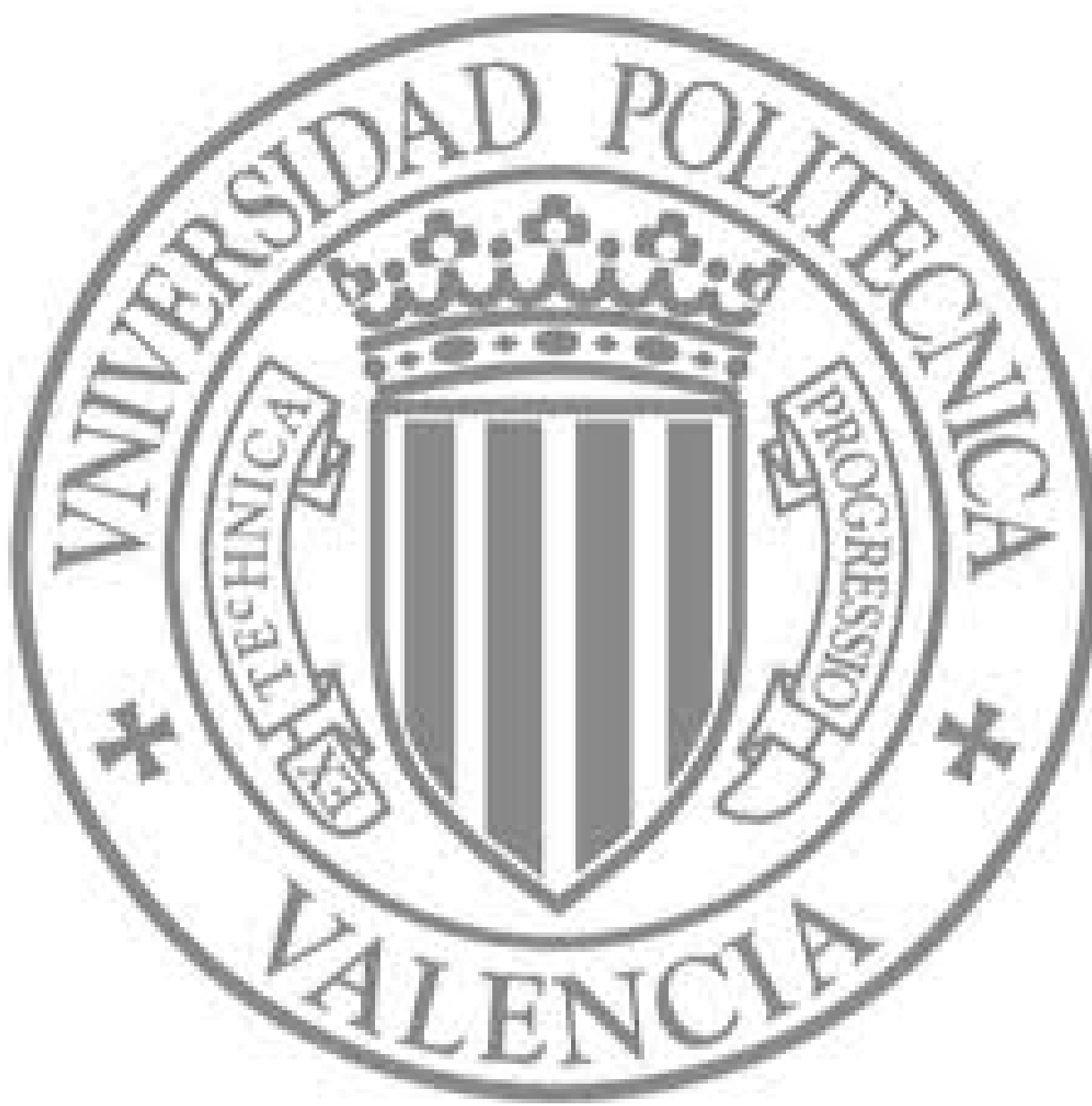

The final publication is available at

http://dx.doi.org/10.1016/j.fss.2012.11.001

Copyright Elsevier 


\title{
Fuzzy quasi-metrics for the Sorgenfrey line
}

\author{
Valentín Gregori ${ }^{\mathrm{a}, 1, *}$, Samuel Morillas ${ }^{\mathrm{a}, 1}$, Bernardino Roig ${ }^{\mathrm{b}}$ \\ ${ }^{a}$ Instituto Universitario de Matemática Pura y Aplicada, Universidad Politécnica de \\ Valencia, Camino de Vera s/n, 46022 Valencia, Spain. \\ ${ }^{b}$ Instituto para la Gestión Integrada de Zonas Costeras, Universidad Politécnica de \\ Valencia, C/Paranimf 1, 46730 Gandia, Spain.
}

\begin{abstract}
We endow the set of real numbers with a family of fuzzy quasi-metrics, in the sense of George and Veeramani, which are compatible with the Sorgenfrey topology. Although these fuzzy quasi-metrics are not deduced explicitly from a quasi-metric, they possess interesting properties related to completeness. For instance, we prove that they are balanced and complete in the sense of Doitchinov and that only one of them is right K-sequentially complete. We also observe that compatible fuzzy quasi-metrics for the Sorgenfrey line cannot be left (weakly right) K-sequentially complete.
\end{abstract}

Keywords: Fuzzy quasi-metric space, Sorgenfrey line, Cauchy sequence, left (right) K-Cauchy sequence.

\section{Introduction}

Probabilistic-quasi-pseudo-metric spaces (briefly, PqpM-spaces) are a natural generalization of probabilistic metric spaces (PM-spaces) due to Menger [24], and recently some aspects on them have been studied by Cho, Grabiec and Saadati in [3, 9, 10]. Kramosil and Michalek [21] gave a notion of fuzzy metric space which could be considered as a reformulation, in the fuzzy context, of the notion of PM-space. Later, George and Veeramani introduced and studied $[6,8]$ a notion of fuzzy metric space which is a slight modification of the one given by Kramosil and Michalek, but in this case it cannot be regarded, in general, as a Menger space [14]. From now on, by

\footnotetext{
${ }^{*}$ Corresponding author:

Email address: vgregori@mat.upv.es (Valentín Gregori)

${ }^{1}$ The first and second authors acknowledge the support of Spanish Ministry of Education and Science under grant MTM 2009-12872-C02-01.
} 
fuzzy metric we mean a fuzzy metric in the sense of George and Veeramani. Several authors have contributed to the development of this theory, for instance $[15,16,17,34,30,32,25,26]$. In particular, in $[15,7]$ the authors proved that the class of topological spaces that are fuzzy metrizable agrees with the class of metrizable spaces. It is interesting to notice that these fuzzy metrics have been successfully used in color image filtering $[27,13,2]$.

In [28] J.H. Park extended the notion of fuzzy metric to the intuitionistic context. On the other hand, in [18] the authors introduced the concept of fuzzy quasi-(pseudo-)metric that, in a similar way to the classical theory [5], generalizes the concept of fuzzy metric due to George and Veeramani. Obviously, fuzzy quasi-pseudo-metrics cannot be regarded, in general, as PqpM-spaces. Nevertheless (as in the case of fuzzy metrics with respect to PM-spaces), some concepts and results related to fuzzy quasi-pseudo-metric spaces are similar to their corresponding ones in PqpM-spaces. This is the case of the different concepts of sequential completeness studied in [10].

Many authors have contributed to the study of completeness and completion of quasi-metrics based on the notion of Cauchy sequence, for instance $[36,20,29,33,4,1,31,22,23]$, and in some cases this study has been extended to the fuzzy context $[12,19]$. Obviously, these studies are particularly interesting in the case of quasi-metrizable non metrizable topological spaces.

It is well known that the Sorgenfrey line provides a relevant example of a quasi-metrizable space which is not metrizable. So, this space is fuzzy quasimetrizable but not fuzzy metrizable [15], [18]). Customarily, every fuzzy metric deduced from a metric $d$ ([13], Section 3.2) inherits the completeness properties of $d$, as it is the case of the standard fuzzy-metric. In that case, the study of such properties is not relevant, in general. Further, many properties based on the t-parameter are fulfilled by this type of fuzzy metrics (for instance, the standard fuzzy metric is completable [16, 17], principal [11], strong (non-Archimedean) [14], ...), and so the construction of fuzzy metrics not deduced explicitly from a metric turns interesting, in order to solve fuzzy metric questions. These facts hold in the fuzzy quasi-metric framework (Remark 5.14).

For the reasons above we here endow the Sorgenfrey line with a family of fuzzy quasi-metrics which are not deduced explicitly from a quasi-metric and we focus our attention on some aspects of their completeness. Then, we study the (sequential) completeness of these fuzzy quasi-metrics in the sense of Doitchinov [4, 12] and the different notions of Cauchy sequence in [29]. We prove that all these fuzzy quasi-metrics are balanced and complete in the sense of Doichinov. On the other hand, if $M$ is a compatible fuzzy 
quasi-metric with the Sorgenfrey line on $\mathbb{R}$, then we prove that $M$ is not left $\mathrm{K}$-sequentially complete nor weakly right $\mathrm{K}$-sequentially complete, using results of [31] and [1], respectively. We illustrate these facts with appropriate examples and we prove that only one of our family of fuzzy quasi-metrics is right K-sequentially complete, Theorem 5.10. We also introduce, in a natural way, the concept of uniform equivalence between fuzzy quasi-metrics defined on the same set and study some properties. Then we prove that all members of our family of fuzzy quasi-metrics on $\mathbb{R}$, excepting those mentioned in Theorem 5.10, are uniformly equivalent.

The structure of the paper is as follows. After the preliminary section, we introduce a family of fuzzy quasi-metrics which are compatible with the Sorgenfrey line, in Section 3. In Section 4 we prove that all of them are balanced and complete, both in the sense of Doitchinov. In Section 5 we prove that only one of the mentioned fuzzy quasi-metrics is right Ksequentially complete, and in Section 6 we show that the rest of fuzzy quasimetrics are uniformly equivalent.

\section{Preliminaries}

From now on, we will denote by $\mathbb{N}$ and $\mathbb{R}$ the set of positive integers and real numbers, respectively. Our basic reference for general topology is [5].

Let us recall [35] that a continuous $t$-norm is a binary operation $*$ : $[0,1] \times[0,1] \rightarrow[0,1]$ such that $([0,1], \leq, *)$ is an ordered Abelian topological monoid with unit 1 .

Definition 2.1. [18] A fuzzy quasi-metric space is an ordered triple $(X, M, *)$ such that $X$ is a (nonempty) set, $*$ is a continuous t-norm and $M$ is a fuzzy set on $X \times X \times] 0,+\infty[$ satisfying the following conditions, for all $x, y, z \in X, s, t>0$ :

Q1: $M(x, y, t)>0$

Q2: $M(x, y, t)=1$ if and only if $x=y$

Q3: $M(x, y, t) * M(y, z, s) \leq M(x, z, t+s)$

Q4: $M(x, y, \cdot):] 0,+\infty[\rightarrow] 0,1]$ is continuous.

Condition $Q 2$ is equivalent to the following:

$M(x, x, t)=1$ for all $x \in X$ and $t>0$, and $M(x, y, t)<1$ for all $x \neq y$ and $t>0$.

If $(X, M, *)$ is a fuzzy quasi-metric space, we will say that $(M, *)$, or simply $M$, is a fuzzy quasi-metric on $X$. A fuzzy quasi-metric $M$ is a fuzzy 
metric, in the sense of George and Veeramani [6], if $M(x, y, t)=M(y, x, t)$ for all $x, y \in X, t>0$.

Frequently we will use the following well-known fact without mention: $M(x, y, \cdot)$ is nondecreasing for all $x, y \in X$.

Example 2.2. Let $(X, d)$ be a quasi-metric space [5]. Define $a * b=a b$ for all $a, b \in[0,1]$, and let $M_{d}$ be the fuzzy set on $\left.X \times X \times\right] 0,+\infty[$ defined by

$$
M_{d}(x, y, t)=\frac{t}{t+d(x, y)}
$$

Then $\left(X, M_{d}, \cdot\right)$ is a fuzzy quasi-metric space and $M_{d}$ is called the standard fuzzy quasi-metric induced by d (see [18], [6]). Notice that $\left(X, M_{d}, \wedge\right)$ is also a fuzzy quasi-metric space, where $\wedge$ denotes the $t$-norm min.

Every fuzzy quasi-metric $M$ on $X$ generates a $T_{1}$-topology $\tau_{M}$ on $X$ which has as a base the family of open sets of the form $\left\{B_{M}(x, r, t): x \in\right.$ $X, r \in] 0,1[, t>0\}$, where $B_{M}(x, r, t)=\{y \in X: M(x, y, t)>1-r\}$ for all $r \in] 0,1[$.

If $(X, d)$ is a quasi-metric space, then the topology $\tau(d)$ generated by $d$ coincides with the topology $\tau_{M_{d}}$ generated by the standard fuzzy quasimetric $M_{d}$.

As in the classical case we say that a topological space $(X, \tau)$ is fuzzy quasi-metrizable if there is a fuzzy quasi-metric $M$ on $X$ such that $\tau=\tau_{M}$. In such a case $M$ is said to be compatible with $\tau$, and also that $\tau$ admits a (compatible) fuzzy quasi-metric. From the above result it follows that every quasi-metrizable topological space admits a compatible fuzzy quasi-metric. Conversely, if $(X, M)$ is a fuzzy quasi-metric space, the family $\left\{U_{n}: n \in \mathbb{N}\right\}$ where $U_{n}=\left\{(x, y) \in X \times X: M\left(x, y, \frac{1}{n}\right)>1-\frac{1}{n}\right\}$ is a countable base for a quasi-uniformity $\mathcal{U}_{M}$ on $X$ compatible with $\tau_{M}$ [18]. Hence, the topological space $\left(X, \tau_{M}\right)$ is quasi-metrizable [5].

Proposition 2.3. A sequence $\left\{x_{n}\right\}$ in a fuzzy quasi-metric space $(X, M, *)$ converges to $x$ if and only if $\lim _{n} M\left(x, x_{n}, t\right)=1$ for all $t>0$.

Definition 2.4. A fuzzy quasi-metric $M$ is called stationary [17] if $M$ does not depend on $t$. In this case we will write $M(x, y)$ instead of $M(x, y, t)$.

\section{Introducing the family of fuzzy quasi-metrics}

We start this section introducing a family of fuzzy quasi-metrics on $\mathbb{R}$. 
Proposition 3.1. Let $k \geq 0$ and consider the fuzzy set $N_{k}$ on $\left.\mathbb{R}^{2} \times\right] 0, \infty[$ defined by

$$
N_{k}(x, y, t)=\left\{\begin{array}{lll}
\frac{e^{x}+k}{e^{y}+k} & \text { if } & x \leq y \\
\frac{1}{2} & \text { if } & x>y
\end{array}\right.
$$

Then $\left(\mathbb{R}, N_{k}, \cdot\right)$ is a stationary fuzzy quasi-metric space.

Proof. It is straightforward.

From now on, as usual, we will write $N_{k}(x, y)$ instead of $N_{k}(x, y, t)$.

Proposition 3.2. Consider the fuzzy set $N$ on $\left.\mathbb{R}^{2} \times\right] 0, \infty[$ defined by

$$
N(x, y, t)=\left\{\begin{array}{lll}
\frac{e^{x}+t}{e^{y}+t} & \text { if } \quad x \leq y \\
\frac{1}{2} & \text { if } \quad x>y .
\end{array}\right.
$$

Then $(\mathbb{R}, N, \cdot)$ is a fuzzy quasi-metric space.

Proof. We only prove the triangle inequality.

Notice that for $x, y \in \mathbb{R}$ and $t>0$ we have that $N(x, y, t)=N_{t}(x, y)$, where $N_{t}$ is the stationary fuzzy quasi-metric of Proposition 3.1. Now, the family $\left\{N_{t}: t>0\right\}$ is increasing, in the sense that $N_{t} \leq N_{s}$ if $t<s$. Then for $x, y, z \in \mathbb{R}$ and $t, s>0$ we have

$$
\begin{aligned}
N(x, z, t+s) & =N_{t+s}(x, z) \geq N_{t+s}(x, y) \cdot N_{t+s}(y, z) \geq N_{t}(x, y) \cdot N_{s}(y, z) \\
& =N(x, y, t) \cdot N(y, z, s) .
\end{aligned}
$$

Throughout the paper $N_{t}(t \geq 0)$ and $N$ will denote the fuzzy quasimetrics of Proposition 3.1 and 3.2, respectively.

Remark 3.3. We have just seen in the proof of Proposition 3.2 that $N$ can be defined by the family $\left\{N_{t}: t>0\right\}$. Also, $N_{0}$, which will take an interesting role in Section 5, can be defined by this family. Indeed, $N_{0}=\inf \left\{N_{t}: t>0\right\}$.

It is easy to verify that $(\mathbb{R}, N, \wedge)$ is not a fuzzy quasi-metric space, so $N$ is not a standard fuzzy quasi-metric.

From now on we will denote by $\mathcal{S}$ the Sorgenfrey topology (also known as the right half-open interval topology) on $\mathbb{R}$.

\section{Proposition 3.4.}

(i) $\tau_{N_{k}}=\mathcal{S}$ for all $k \geq 0$.

(ii) $\tau_{N}=\mathcal{S}$. 
Proof. (i) For $k \geq 0$ the open balls centered at $x \in \mathbb{R}$ with radius $r \in] 0, \frac{1}{2}$ [ in $\left(\mathbb{R}, N_{k}, \cdot\right)$ are of the form

$$
B_{N_{k}}(x, r)=\left[x, \ln \frac{e^{x}+k r}{1-r}[\right.
$$

and clearly $\tau_{N_{k}}$ is the topology $\mathcal{S}$ on $\mathbb{R}$.

(ii) It is easy to verify that $\tau_{N}=\bigvee_{t>0} \tau_{N_{t}}$ and hence, by $(i), \tau_{N}=\mathcal{S}$.

\section{D-completeness}

Let $a_{n m} \in \mathbb{R}$ for $n, m=1,2, \ldots$ We write $\lim _{n, m} a_{n, m}=r$ if for each $\varepsilon>0$ there exists $n_{\varepsilon} \in \mathbb{N}$ such that $\left|a_{n m}-r\right|<\varepsilon$ for all $n, m \geq n_{\varepsilon}$.

In [4], Doitchinov constructed a completion theory for a type of quasimetrics called balanced. He pointed out that the quasi-metric $d$ on $\mathbb{R}$ given by

$$
d(x, y)= \begin{cases}y-x & \text { if } x \leq y \\ 1 & \text { if } x>y\end{cases}
$$

which generates the Sorgenfrey topology on $\mathbb{R}$, is balanced and complete in that theory.

According to Doitchinov's completion theory, in [12] the authors introduced the next concepts.

Definition 4.1. Let $(X, M, *)$ be a fuzzy quasi-metric space. The sequence $\left\{x_{n}\right\}$ in $X$ is D-Cauchy if there exists a sequence $\left\{y_{n}\right\}$ in $X$ such that

$$
\lim _{m, n}\left(y_{m}, x_{n}, t\right)=1 \text { for all } t>0
$$

Any sequence $\left\{y_{m}\right\}$ satisfying the above condition with respect to a Cauchy sequence $\left\{x_{n}\right\}$ will be called a consequence to $\left\{x_{n}\right\}$.

Definition 4.2. A fuzzy quasi-metric space $(X, M, *)$ is called D-complete, or simply $M$ is D-complete, if every $D$-Cauchy sequence in $X$ is convergent with respect to $\tau_{M}$.

Definition 4.3. Let $(X, M, *)$ be a fuzzy quasi-metric space. The fuzzy quasi-metric $M$ is called balanced or BF-quasi-metric if it satisfies the following condition:

(BF) If $\left\{x_{n}^{\prime}\right\}$ and $\left\{x_{m}^{\prime \prime}\right\}$ are sequences in $X, x^{\prime}, x^{\prime \prime} \in X, t_{1}, t_{2}>0$, then from $M\left(x^{\prime}, x_{n}^{\prime}, t_{1}\right) \geq r^{\prime}$ for each $n, M\left(x_{m}^{\prime \prime}, x^{\prime \prime}, t_{2}\right) \geq r^{\prime \prime}$ for each $m$, and $\lim _{m, n} M\left(x_{m}^{\prime \prime}, x_{n}^{\prime}, t\right)=1$ for all $t>0$ it follows that $M\left(x^{\prime}, x^{\prime \prime}, t_{1}+t_{2}\right) \geq r^{\prime} * r^{\prime \prime}$. 
Proposition 4.4. $\left(\mathbb{R}, N_{k}, \cdot\right)$ is balanced for each $k \geq 0$.

Proof. Fix $k \geq 0$. Let $\left\{x_{n}^{\prime}\right\}$ and $\left\{x_{m}^{\prime}\right\}$ be two sequences in $\left.\mathbb{R}, r^{\prime}, r^{\prime \prime} \in\right] 0,1[$, $x^{\prime}, x^{\prime \prime} \in \mathbb{R}$ such that $N_{k}\left(x^{\prime}, x_{n}^{\prime}\right) \geq r^{\prime}$ and $N_{k}\left(x_{m}^{\prime \prime}, x^{\prime \prime}\right) \geq r^{\prime \prime}$ for all $m, n \in \mathbb{N}$ and $\lim _{m, n} N_{k}\left(x_{m}^{\prime \prime}, x_{n}^{\prime}\right)=1$.

From $\lim _{n, m} N_{k}\left(x_{m}^{\prime \prime}, x_{n}^{\prime}\right)=1$ we have that there exists $n_{0} \in \mathbb{N}$ such that $N_{k}\left(x_{m}^{\prime \prime}, x_{n}^{\prime}\right)>\frac{1}{2}$ for all $m, n \geq n_{0}$, hence by definition of $N_{k}$ we have that $x_{m}^{\prime \prime}<x_{n}^{\prime}$ for all $m, n \geq n_{0}$.

Since $\left\{x_{m}^{\prime \prime}\right\}$ is upper bounded and $\left\{x_{n}^{\prime}\right\}$ is lower bounded we have that $c^{\prime \prime}=\sup \left\{x_{m}^{\prime \prime}: m \geq n_{0}\right\} \leq \inf \left\{x_{n}^{\prime}: n \geq n_{0}\right\}=c^{\prime}$.

Suppose $c^{\prime \prime}<c^{\prime}$. Then by definition of $N_{k}$ we have $N_{k}\left(x_{m}^{\prime \prime}, x_{n}^{\prime}\right) \leq$ $N_{k}\left(c^{\prime \prime}, c^{\prime}\right)=\alpha<1$. Hence, $N_{k}\left(x_{m}^{\prime \prime}, x_{n}^{\prime}\right) \leq \alpha$ for all $m, n \geq n_{0}$, a contradiction. Thus $c^{\prime \prime}=c^{\prime}$. Put $\sup \left\{x_{m}^{\prime \prime}: m \geq n_{0}\right\}=c=\inf \left\{x_{n}^{\prime}: n \geq n_{0}\right\}$. We will show that $N_{k}\left(c, x^{\prime \prime}\right) \geq r^{\prime \prime}$. We distinguish two cases:

(a) First, suppose $r^{\prime \prime}>\frac{1}{2}$. Since $N_{k}\left(x_{m}^{\prime \prime}, x^{\prime \prime}\right)>\frac{1}{2}$ then by definition of $N_{k}$ we have $x_{m}^{\prime \prime}<x^{\prime \prime}$ for all $m \in \mathbb{N}$ and also $c \leq x^{\prime \prime}$. Hence $N_{k}\left(c, x^{\prime \prime}\right) \geq$ $N_{k}\left(x_{n_{0}}^{\prime \prime}, x^{\prime \prime}\right) \geq r^{\prime \prime}$.

(b) Second, suppose $r^{\prime \prime} \leq \frac{1}{2}$. We distinguish two cases:

(b1) Suppose $c>x^{\prime \prime}$. Then by definition of $N_{k}$ we have $N_{k}\left(c, x^{\prime \prime}\right)=$ $\frac{1}{2} \geq r^{\prime \prime}$.

(b2) Suppose $c \leq x^{\prime \prime}$. Then $x_{m}^{\prime \prime} \leq x^{\prime}$ for all $m \geq n_{0}$ and then, by definition of $N_{k}, N_{k}\left(c, x^{\prime \prime}\right) \geq N_{k}\left(x_{n_{0}}^{\prime \prime}, x^{\prime \prime}\right) \geq r^{\prime \prime}$.

In a similar way it can be proved that $N_{k}\left(x^{\prime}, c\right) \geq r^{\prime}$.

Finally, $N_{k}\left(x^{\prime}, x^{\prime \prime}\right) \geq N_{k}\left(x^{\prime}, c\right) \cdot N_{k}\left(c, x^{\prime \prime}\right) \geq r^{\prime} \cdot r^{\prime \prime}$.

Proposition 4.5. $(\mathbb{R}, N, \cdot)$ is balanced.

Proof. Let $\left\{x_{n}^{\prime}\right\}$ and $\left\{x_{m}^{\prime \prime}\right\}$ be sequences in $\left.\mathbb{R}, r^{\prime}, r^{\prime \prime} \in\right] 0,1\left[, t_{1}, t_{2}>0\right.$, $x^{\prime}, x^{\prime \prime} \in \mathbb{R}$ such that $N\left(x^{\prime}, x_{n}^{\prime}, t_{1}\right) \geq r^{\prime}, N\left(x_{m}^{\prime \prime}, x^{\prime \prime}, t_{2}\right) \geq r^{\prime \prime}$ for all $n, m \in \mathbb{N}$ and $\lim _{m, n} N\left(x_{m}^{\prime \prime}, x_{n}^{\prime}, t\right)=1$ for all $t>0$.

We have that $N\left(x^{\prime}, x_{n}^{\prime}, t_{1}+t_{2}\right) \geq r^{\prime}, N\left(x_{m}^{\prime \prime}, x^{\prime \prime}, t_{1}+t_{2}\right) \geq r^{\prime \prime}$ for all $n, m \in \mathbb{N}$, i.e. $N_{t_{1}+t_{2}}\left(x^{\prime}, x_{n}^{\prime}\right) \geq r^{\prime}, N_{t_{1}+t_{2}}\left(x_{m}^{\prime \prime}, x^{\prime \prime}\right) \geq r^{\prime \prime}$ for all $n, m \in \mathbb{N}$ and also $\lim _{m, n} N_{t_{1}+t_{2}}\left(x_{m}^{\prime \prime}, x_{n}^{\prime}\right)=1$. Now, by the previous proposition $N_{t_{1}+t_{2}}$ is balanced, so, we have that $N_{t_{1}+t_{2}}\left(x^{\prime}, x^{\prime \prime}\right) \geq r^{\prime} \cdot r^{\prime \prime}$, i.e. $N\left(x^{\prime}, x^{\prime \prime}, t_{1}+t_{2}\right) \geq$ $r^{\prime} \cdot r^{\prime \prime}$. 
We omit the proof of the next proposition.

Proposition 4.6. If $\left\{x_{n}\right\}$ is a convergent sequence in a fuzzy quasi-metric space $(X, M, *)$ then $\left\{x_{n}\right\}$ is D-Cauchy.

Proposition 4.7. $\left(\mathbb{R}, N_{k}, \cdot\right)$ is D-complete for all $k \geq 0$.

Proof. Fix $k \geq 0$. Let $\left\{x_{n}\right\}$ be a D-Cauchy sequence in $\left(\mathbb{R}, N_{k}, \cdot\right)$ and let $\left\{y_{m}\right\}$ a cosequence to $\left\{x_{n}\right\}$, so $\lim _{m, n} N_{k}\left(y_{m}, x_{n}\right)=1$. Then for $\varepsilon=\frac{1}{2}$ there exists $p_{2} \geq 2$ such that $N_{k}\left(y_{m}, x_{s}\right)>1-\frac{1}{2}$ for all $m, s \geq p_{2}$, and so $y_{m} \leq x_{s}$ for all $s \geq p_{2} \geq 2$.

Now by induction we construct a sequence $\left\{y_{p_{m}}\right\}$ such that $p_{m} \geq m$, $p_{m} \geq p_{m-1}$ and $N_{k}\left(y_{m}, x_{s}\right)>1-\frac{1}{m}$ for all $m, s \geq p_{m}$. So, $y_{m} \leq x_{s}$ for all $m, s \geq p_{m}$, and in particular, $N_{k}\left(y_{p_{m}}, x_{s}\right)>1-\frac{1}{m}$ for all $s \geq p_{m}$.

Clearly it is satisfied that $a=\sup \left\{y_{m}: m \geq p_{2}\right\} \leq b=\inf \left\{x_{m}: m \geq\right.$ $\left.p_{2}\right\}$. We will see that $a=b$. Indeed, in other case, it should be $a<b$. Hence $N_{k}(a, b)=\alpha<1$ and so $\lim _{m, n} N_{k}\left(y_{m}, x_{n}\right) \leq N_{k}(a, b)<1$, a contradiction.

We will see that $\left\{x_{n}\right\}$ converges to $b$ in $\mathcal{S}$. For it, let $\left.\varepsilon \in\right] 0, \frac{1}{2}[$ and take the basic neighborhood $[b, b+\varepsilon[$ of $b$.

We consider the decreasing function $f:] 1, \infty[\rightarrow \mathbb{R}$ defined by $f(n)=$ $\ln \frac{e^{b}+\frac{k}{n}}{1-\frac{1}{n}}$. Clearly $f$ is continuous on $n$, when considering the usual topology of $\mathbb{R}$, and it satisfies $f(n)>b$ for all $n>1$ and also $\lim _{n \rightarrow \infty} f(n)=b$. Therefore we can choose $n_{0} \in \mathbb{N}$ such that $\ln \frac{e^{b}+\frac{k}{n_{0}}}{1-\frac{1}{n_{0}}}<b+\varepsilon$.

Now, by construction of $\left\{y_{p_{m}}\right\}$ we have that $N_{k}\left(y_{p_{n_{0}}}, x_{n}\right)>1-\frac{1}{n_{0}}$ for all $n \geq p_{n_{0}}$, and therefore $N_{k}\left(b, x_{n}\right) \geq N_{k}\left(y_{p_{n_{0}}}, x_{n}\right)>1-\frac{1}{n_{0}}$ for all $n \geq p_{n_{0}}$.

Hence, $\frac{e^{b}+k}{e^{x_{n}}+k}>1-\frac{1}{n_{0}}$ for all $n \geq p_{n_{0}}$ and then, after an easy computation, we have that $x_{n}<\ln \frac{e^{b}+\frac{k}{n_{0}}}{1-\frac{1}{n_{0}}}<b+\varepsilon$ for all $n \geq p_{n_{0}}$.

Theorem 4.8. $(\mathbb{R}, N, \cdot)$ is D-complete.

Proof. Let $\left\{x_{n}\right\}$ be a D-Cauchy sequence in $(\mathbb{R}, N, \cdot)$. By Definition $4.1\left\{x_{n}\right\}$ is also D-Cauchy in $\left(\mathbb{R}, N_{t}, \cdot\right)$ for each $t>0$. Then by Proposition 4.7 and 3.4 the sequence $\left\{x_{n}\right\}$ converges with respect to $\mathcal{S}$. 
Remark 4.9. If $\left\{y_{m}\right\}$ is a cosequence of the Cauchy sequence $\left\{x_{n}\right\}$ in $\left(\mathbb{R}, N_{k}, \cdot\right)$ for some $k>0$ then $\left\{y_{m}\right\}$ is a cosequence of $\left\{x_{n}\right\}$ in $\left(\mathbb{R}, N_{t}, \cdot\right)$ for all $t>0$.

Indeed, in Proposition 4.7 we have proved that $\left\{x_{n}\right\}$ converges to $b \in \mathbb{R}$ in $\mathcal{S}$, and hence $\left\{x_{n}\right\}$ converge to $b$ in the usual topology of $\mathbb{R}$, i.e. $\lim _{n} x_{n}=b$. Now, from $\lim _{m, n} N_{k}\left(y_{m}, x_{n}\right)=1$ it holds $\lim _{m, n} \frac{e^{y_{m}}+k}{e^{x_{n}}+k}=1$, so we conclude that $\lim _{m}\left(e^{y_{m}}+k\right)=e^{b}+k$. Therefore $\lim _{m} y_{m}=b$ and $\lim _{m, n} \frac{e^{y_{m}}+t}{e^{x_{n}}+t}=1$ for all $t>0$, and so $\left\{y_{m}\right\}$ is a cosequence of $\left\{x_{n}\right\}$ in $\left(\mathbb{R}, N_{t}, \cdot\right)$ for all $t>0$.

\section{Fuzzy left (right) K-sequentially completeness}

In [29], the authors reviewed and introduced some definitions on completeness in quasi-pseudo-metric spaces. Next, we adapt the definitions (iv)(vi) to our fuzzy context.

Definition 5.1. Let $(X, M, *)$ be a fuzzy quasi-metric space. A sequence $\left\{x_{n}\right\}$ in $X$ is said to be

(a) right (left) $K$-Cauchy if for each $\varepsilon \in] 0,1[$ and $t>0$ there is an integer $k$ such that $M\left(x_{r}, x_{s}, t\right)>1-\varepsilon$ for $r \geq s \geq k(s \geq r \geq k)$.

(b) weakly left (right) $K$-Cauchy if for each $\varepsilon \in] 0,1[$ and $t>0$ there is an integer $k$ such that $M\left(x_{k}, x_{m}, t\right)>1-\varepsilon\left(M\left(x_{m}, x_{k}, t\right)>1-\varepsilon\right)$ for all $m \geq k$.

In the same way it is possible to adapt definitions (i)-(ii) of [29] to our fuzzy context but the reader will notice after seeing Corollary 5.7 that they would not be really relevant in the scope of this paper.

Attending to Definition 5.1 we obtain the next proposition.

Proposition 5.2. Let $\left\{x_{n}\right\}$ be a sequence in $\mathbb{R}$. Then $\left\{x_{n}\right\}$ is [weakly] left (right) Cauchy in $(\mathbb{R}, N, \cdot)$ iff $\left\{x_{n}\right\}$ is [weakly] left (right) Cauchy in $\left(\mathbb{R}, N_{t}, \cdot\right)$ for all $t>0$.

Corresponding to the above four definitions of Cauchy sequence we have the following four notions of completeness.

Definition 5.3. Let $(X, M, *)$ be a fuzzy quasi-metric space. Then $X$ is said to be [weakly] left (right) $K$-sequentially complete if every [weakly] left (right) $K$-Cauchy sequence in $X$ converges to some point in $X$ with respect to $\tau_{M}$. 
Since left (right) K-Cauchy sequences are weakly left (right) K-Cauchy then weakly left (right) K-sequential completeness implies left (right) Ksequential completeness. The reader may find further interesting results related to completeness, but in the context of PqpM-spaces, in [10].

Next, we will study the completeness of $N_{t}(t \geq 0)$ and $N$, in the context of this section.

Definition 5.4. A topological space $(X, \tau)$ is called completely left K-sequentially fuzzy quasi-metrizable if it admits a left $K$-sequentially complete fuzzy quasimetric $M$ which is compatible with $\tau$, i.e. $\tau_{M}=\tau$. This terminology is extended, in a natural way, to the other completeness concepts.

Theorem 5.5. Let $(X, M, *)$ be a weakly right $K$-sequentially complete fuzzy quasi-metric space. Then $\left(X, \tau_{M}\right)$ is completely weakly right K-sequentially quasi-metrizable.

Proof. It is easy to verify that the countable family $\left\{U_{n}: n \in \mathbb{N}\right\}$ where $U_{n}=\left\{(x, y) \in X \times X: M\left(x, y, \frac{1}{n}\right)>1-\frac{1}{n}\right\}$ is a base for a quasi-uniformity $U_{M}$. Then, since $\tau_{M}$ is $T_{1}$ there exists a quasi-metric $d$ on $X$ whose induced quasi-uniformity coincides with $U_{M}$. Imitating the proof of [15] Theorem 2 one can easily obtain that $d$ is a weakly right $\mathrm{K}$-sequentially complete quasi-metric on $X$.

In the same way it is proved the next theorem.

Theorem 5.6. Let $(X, M, *)$ be a left $K$-sequentially complete fuzzy quasimetric space. Then $\left(X, \tau_{M}\right)$ is completely left $K$-sequentially quasi-metrizable.

It is known that the Sorgenfrey line does not admit any weakly right Ksequentially complete quasi-metric [1] nor any left K-sequentially complete quasi-metric [31]. Then, by Theorem 5.5 and 5.6 we have the next corollary.

Corollary 5.7. The Sorgenfrey line does not admit any weakly right $K$ sequentially complete fuzzy-quasi-metric, nor any a left K-sequentially complete fuzzy quasi-metric.

Proposition 5.8. $\left(\mathbb{R}, N_{t}, \cdot\right)$ is not right $K$-sequentially complete for each $t>0$.

Proof. Fix $t>0$. Consider the sequence $\left\{x_{n}\right\}$ where $x_{n}=-n$ for each $n \in \mathbb{N}$. We will see that $\left\{x_{n}\right\}$ is right $\mathrm{K}$-Cauchy in $\left(\mathbb{R}, N_{t}, \cdot\right)$. 
Let $\varepsilon \in] 0, \frac{1}{2}[$. Consider the decreasing function $f: \mathbb{R} \rightarrow] 0,1[$ given by $f(k)=\frac{t}{e^{k}+t}$. Obviously, $f$ is continuous on $k$, in the usual topology of $\mathbb{R}$, and $\lim _{k \rightarrow-\infty} f(k)=1$, so we can find $k \in \mathbb{N}$ such that $\frac{t}{e^{-k}+t}>1-\varepsilon$. Now, for $r \geq s \geq k$ we have

$$
M\left(x_{r}, x_{s}, t\right)=\frac{e^{x_{r}}+t}{e^{x_{s}}+t}=\frac{e^{-r}+t}{e^{-s}+t}>\frac{t}{e^{-k}+t}>1-\varepsilon
$$

and hence $\left\{x_{n}\right\}$ is right K-Cauchy in $\left(\mathbb{R}, N_{t}, \cdot\right)$.

Finally, $\left\{x_{n}\right\}$ does not converge in $\mathbb{R}$ with respect to $\mathcal{S}$. Indeed, for each $x \in \mathbb{R}$ there exists $k \in \mathbb{N}$ such that $-k<x$, then $N_{t}\left(x, x_{n}\right)=\frac{1}{2}$ for all $n \geq k$, and $\left\{x_{n}\right\}$ does not converge to $x$.

By Proposition 5.2 the sequence $\left\{x_{n}\right\}$ of Proposition 5.8 is also right $\mathrm{K}$-Cauchy in $(\mathbb{R}, N, \cdot)$ and, as we have seen, it does not converge in $\mathcal{S}$. So we have the next corollary.

Corollary 5.9. $(\mathbb{R}, N, \cdot)$ is not right $K$-sequentially complete.

Theorem 5.10. $\left(\mathbb{R}, N_{0}, \cdot\right)$ is right K-sequentially complete.

Proof. Let $\left\{x_{n}\right\}$ be a right K-Cauchy sequence in $\mathbb{R}$. So, for $\left.\varepsilon \in\right] 0, \frac{1}{2}[$ there exists $k \in \mathbb{N}$ such that $N_{0}\left(x_{r}, x_{s}\right)>1-\frac{1}{2}$ for $r \geq s \geq k$. Consequently $e^{x_{r}} \leq e^{x_{s}}$ for $r \geq s \geq k$ and hence (we can suppose that) $\left\{x_{n}\right\}$ is decreasing. We will see that $\left\{x_{n}\right\}$ is lower bounded in the usual metric of $\mathbb{R}$.

Indeed, we have that $M_{0}\left(x_{r}, x_{k}\right)>\frac{1}{2}$ for all $r \geq k$ and it means that $\frac{e^{x_{r}}}{e^{x_{k}}}>\frac{1}{2}$ and so it is easy to verify that $\left.x_{r} \in\right] x_{k}+\ln \frac{1}{2}, x_{k}[$ for all $r \geq k$ and thus $\left\{x_{n}\right\}$ is lower bounded. Let $c=\inf \left\{x_{r}: r \geq k\right\}$. The sequence $\left\{x_{n}\right\}$ converges to $c$ in the usual topology of $\mathbb{R}$, and since $\left\{x_{n}\right\}$ is decreasing it converges to $c$ in $\mathcal{S}$.

In the next example, according to Corollary 5.7, we give a left K-Cauchy sequence in $\left(\mathbb{R}, N_{t}, \cdot\right)(t \geq 0)$ and $(\mathbb{R}, N, \cdot)$ which does not converge in the Sorgenfrey topology.

Example 5.11. (a) $\left(\mathbb{R}, N_{t}, \cdot\right)$ is not left $K$-sequentially complete for each $t \geq 0$.

Consider the sequence $\left\{x_{n}\right\}$ in $\mathbb{R}$ where $x_{n}=1-\frac{1}{n}, n=2,3, \ldots$ After a tedious computation one can verify that $\left\{x_{n}\right\}$ is left K-Cauchy in $\left(\mathbb{R}, N_{t}, \cdot\right)$ for all $t \geq 0$. Now, $\left\{x_{n}\right\}$ converges to 1 in the usual topology 
of $\mathbb{R}$, but it does not converge in $\mathcal{S}$ since $[1, \varepsilon[$ does not contain any element of $\left\{x_{n}\right\}$, for $\varepsilon>0$. So, $\left(\mathbb{R}, N_{t}, \cdot\right)$ is not left K-sequentially complete.

(b) $(\mathbb{R}, N, \cdot)$ is not left $K$-sequentially complete.

By Proposition 5.2 the sequence $\left\{x_{n}\right\}$ of $(a)$ is left $K$-Cauchy in $(\mathbb{R}, N, \cdot)$ and, as we have seen, it does not converge in $\mathcal{S}$.

We have seen in Theorem 5.10 that $N_{0}$ is right K-sequentially complete. Now, in the next example, according to Corollary 5.7, we give a weakly right K-Cauchy sequence in $\left(\mathbb{R}, N_{0}, \cdot\right)$ which does not converge in the Sorgenfrey topology.

Example 5.12. $\left(\mathbb{R}, N_{0}, \cdot\right)$ is not weakly right $K$-sequentially complete.

Consider the sequence $\left\{x_{n}\right\}$ in $\mathbb{R}$ given by

$$
x_{n}= \begin{cases}1-\frac{1}{n} & \text { if } n \text { odd } \\ 1 & \text { if } n \text { even } .\end{cases}
$$

One can verify that $\left\{x_{n}\right\}$ is weakly right $K$-Cauchy in $\left(\mathbb{R}, N_{0}, \cdot\right)$. Now, as above, $\left\{x_{n}\right\}$ converges to 1 in the usual topology of $\mathbb{R}$, but it does not converge in $\mathcal{S}$.

Remark 5.13. If we adapt the definition (iii) of [29] Definition 1 to our fuzzy setting, we obtain the usual one if $M$ is a fuzzy metric [6]:

A sequence $\left\{x_{n}\right\}$ in the fuzzy quasi-metric space $(X, M, *)$ is Cauchy if for each $\varepsilon \in] 0,1\left[\right.$ and $t>0$ we can find $n_{0} \in \mathbb{N}$ such that $M\left(x_{n}, x_{m}, t\right)>$ $1-\varepsilon$ for $m, n \geq n_{0}$.

It is obvious that a Cauchy sequence in $\left(\mathbb{R}, N_{t}, \cdot\right), t \geq 0$, or in $(\mathbb{R}, N, \cdot)$ is necessary constant after a certain stage, and so it converges.

Remark 5.14. Consider the standard fuzzy quasi-metric $M_{d}$ deduced of the quasi-metric d given by equation (1). We can also deduce from d the family of stationary fuzzy quasi-metrics $\left\{\left(\left(M_{d}\right)_{t}, \cdot\right): t>0\right\}$, compatible with $\mathcal{S}$, where $\left(M_{d}\right)_{t}$ is defined by

$$
\left(M_{d}\right)_{t}(x, y)=\frac{t}{t+d(x, y)}
$$

It is easy to verify that $M_{d}$ and $\left(M_{d}\right)_{t}$ are all balanced and $D$-complete [12].

Now, the situation with respect to completeness studied in this section is very different. Indeed, first we notice that if we define the fuzzy set $\left(M_{d}\right)_{0}$ 
on $X^{2}$ by $\left(M_{d}\right)_{0}=\inf \left\{\left(M_{d}\right)_{t}: t>0\right\}$ then $\left(M_{d}\right)_{0}$ is not a (stationary) fuzzy quasi-metric, since $\left(M_{d}\right)_{0}(x, y)=0$ for each $x, y \in \mathbb{R}$. Now, as a consequence of that, as it is well known, $d$ is right $K$-sequentially complete, then it is easy to prove that $\left(M_{d}\right)$ and $\left(M_{d}\right)_{t}(t>0)$ are all right $K$-sequentially complete.

\section{Uniformly equivalent fuzzy quasi-metric spaces}

In this section $\left(X, M_{1}, *\right)$ and $\left(Y, M_{2}, \diamond\right)$ will be two fuzzy quasi-metric spaces.

Definition 6.1. Let $f: X \rightarrow Y$ be a mapping. Then $f$ is called uniformly continuous if given $\varepsilon \in] 0,1[$ and $t>0$ we can find $\delta \in] 0,1[$ and $s>0$ such that $M_{2}(x, y, t)>1-\varepsilon$ whenever $M_{1}(x, y, s)>1-\delta$. We also say, as usual, that $f:\left(X, M_{1}, *\right) \rightarrow\left(Y, M_{2}, \diamond\right)$ is uniformly continuous. A uniformly continuous mapping is obviously continuous.

Proposition 6.2. Let $f:\left(X, M_{1}, *\right) \rightarrow\left(Y, M_{2}, \diamond\right)$ be uniformly continuous and let $\left\{x_{n}\right\}$ a sequence in $X$. If $\left\{x_{n}\right\}$ is Cauchy in some sense of Definition 4.1 or 5.1 then $\left\{f\left(x_{n}\right)\right\}$ is Cauchy in the same sense.

Proof. It is straightforward.

Two fuzzy quasi-metrics $M_{1}$ and $M_{2}$ on $X$ are called topologically equivalent if $\tau_{M_{1}}=\tau_{M_{2}}$. By Proposition $3.4 N_{k}(k \geq 0)$ and $N$ are all them topologically equivalent.

According to the classical case we introduce the next definition.

Definition 6.3. Two fuzzy quasi-metrics $M_{1}$ and $M_{2}$ on $X$ are said to be uniformly equivalent if the identity mappings $i:\left(X, M_{1}, *\right) \rightarrow\left(X, M_{2}, \diamond\right)$ and $i:\left(X, M_{2}, \diamond\right) \rightarrow\left(X, M_{1}, *\right)$ are uniformly continuous. In this case $M_{1}$ and $M_{2}$ are, obviously, topologically equivalent; the converse is false (Proposition 6.6).

Proposition 6.4. If $M_{1}$ and $M_{2}$ are two uniformly equivalent fuzzy quasimetrics on $X$ then the set of Cauchy sequences, in any of the above senses, in $\left(X, M_{1}, *\right)$ agrees with the set of Cauchy sequences, in the same sense, in $\left(X, M_{2}, \diamond\right)$.

Proof. It is straightforward.

We will see in Remark 6.8 that the converse of Proposition 6.4 is false.

Proposition 6.5. 
(i) $N_{i}$ and $N_{j}$ are uniformly equivalent on $\mathbb{R}$ for all $i, j>0$.

(ii) $N$ and $N_{i}$ are uniformly equivalent on $\mathbb{R}$ for all $i>0$.

Proof. (i) Fix $t, T>0$ with $t<T$. We will see that $N_{t}$ and $N_{T}$ are uniformly equivalent on $\mathbb{R}$.

Since $N_{T}(x, y)>N_{t}(x, y)$, obviously the identity mapping $i:\left(\mathbb{R}, N_{t}, \cdot\right)$ $\rightarrow\left(\mathbb{R}, N_{T}, \cdot\right)$ is uniformly continuous.

We will see that also $i:\left(\mathbb{R}, N_{T}, \cdot\right) \rightarrow\left(\mathbb{R}, N_{t}, \cdot\right)$ is uniformly continuous.

Let $\varepsilon \in] 0, \frac{1}{2}\left[\right.$, and choose $\delta<\min \left\{\frac{t \varepsilon}{T}, \varepsilon\right\}$ with $\delta>0$.

Then if $N_{T}(x, y)>1-\delta$ we have $\frac{e^{x}+T}{e^{y}+t}>1-\delta$ and, after an easy computation, we obtain

$$
x \leq y<\ln \frac{e^{x}+T \cdot \delta}{1-\delta}
$$

and by the election of $\delta$ we have $x \leq y<\ln \frac{e^{x}+t \varepsilon}{1-\varepsilon}$.

Then $e^{y}<\frac{e^{x}+t \varepsilon}{1-\varepsilon}$ and, after an easy computation, we obtain

$$
\frac{e^{x}+t}{e^{y}+t}>1-\varepsilon,
$$

i.e. $N_{t}(x, y)>1-\varepsilon$, and so $i:\left(\mathbb{R}, N_{T}, \cdot\right) \rightarrow\left(\mathbb{R}, N_{t}, \cdot\right)$ is uniformly continuous.

(ii) The proof of $(i i)$ is obtained imitating the proof of $(i)$.

Based on previous results the next proposition is obvious but we choose to give a proof for adding some more information.

Proposition 6.6. $N_{0}$ is not uniformly equivalent to $N_{t}$ for all $t>0$, on $\mathbb{R}$.

Proof. We have seen in the proof of Proposition 5.8 that the sequence $\{-n\}$ is right Cauchy in $\left(\mathbb{R}, N_{t}, \cdot\right)$ for all $t>0$. Now, this sequence is not lower bounded in the usual metric of $\mathbb{R}$. Then as we have seen in the proof of Theorem 5.10, $\{-n\}$ cannot be right K-Cauchy in $N_{0}$. Hence, by Proposition 6.2 the identity mapping $i:\left(\mathbb{R}, N_{t}, \cdot\right) \rightarrow\left(\mathbb{R}, N_{0}, \cdot\right)$ is not uniformly continuous. 
Using the same argument we obtain the next proposition.

Proposition 6.7. $N_{0}$ is not uniformly equivalent to $N$.

Remark 6.8. Taking into account that $\tau_{N_{t}}=\mathcal{S}$ for all $t \geq 0$ and that $N_{t}$ is $D$-complete for all $t \geq 0$ and that convergent sequences, respect to $\mathcal{S}$, are always D-Cauchy, then the set of D-Cauchy sequences in $\left(\mathbb{R}, N_{t}, \cdot\right)$ for $t>0$ agrees with the set of $D$-Cauchy sequence in $\left(\mathbb{R}, N_{0}, \cdot\right)$. Nevertheless, as we have just seen, $N_{t}$ for $t>0$, is not uniformly equivalent to $N_{0}$.

The same argument remains valid for $N$ with respect to $N_{0}$.

Acknowledgement. The authors are grateful to the referees for their valuable suggestions.

\section{References}

[1] E. Alemany and S. Romaguera, On right K-sequentially complete quasimetric spaces, Acta Math. Hungar. 75 (3) (1997) 267-278.

[2] J. G. Camarena, V. Gregori, S. Morillas, A. Sapena, Two-step fuzzy logic-based method for impulsive noise detection in colour images, Pattern Recognition Letters 31 (2010) 1842-1849.

[3] Y. J. Cho, M. Grabiec and R. Saadati, Topologies generated by probabilistic quasi-pseudo-metrics, Tamsui Oxford Journal of Information and Mathematical Sciences 27 (3) (2011) 303-326.

[4] D. Doitchinov. On completeness in quasi-metric spaces, Top. Appl. 30 (1988) 127-148.

[5] P. Fletcher, W. F. Lindgren, Quasi-uniform Spaces, Marcel Dekker, New York and Basel, 1982.

[6] A. George, P. Veeramani, On some results in fuzzy metric spaces, Fuzzy Sets and Systems 64 (1994) 395-399.

[7] A. George, P. Veeramani, Some theorems in fuzzy metric spaces, J. Fuzzy Math. 3 (1995) 933-940.

[8] A. George and P. Veeramani, On some results of analysis for fuzzy metric spaces, Fuzzy Sets and Systems 90 (1997) 365-368.

[9] M. Grabiec, Y. J. Cho and R. Saadati, Families of quasi-pseudometrics generated by probabilistic quasi-pseudo-metric spaces, Surveys in Mathematics and its Applications 2 (2007) 123-143. 
[10] M. Grabiec, Y. J. Cho and R. Saadati, Completeness and fixed points in probabilistic quasi-pseudo-metric spaces, Bulletin of Statistics \& Economics 2 Number A08 (2008) 39-47.

[11] V. Gregori, A. López-Crevillén, S. Morillas and A. Sapena, On convergence in fuzzy metric spaces, Topology Appl. 156 (2009) 3002-3006.

[12] V. Gregori, J. A. Mascarell and A. Sapena, On completion of fuzzy quasi-metric spaces, Topology Appl. 153 (2005) 886-899.

[13] V. Gregori, S. Morillas, A. Sapena, Examples of fuzzy metrics and applications, Fuzzy Sets and Systems 170 (2011) 95-111.

[14] V. Gregori, S. Morillas and A. Sapena, On a class of completable fuzzy metric spaces, Fuzzy Sets and Systems 161 (2010) 2193-2205.

[15] V. Gregori, S. Romaguera, Some properties of fuzzy metric spaces, Fuzzy Sets and Systems 115 (2000) 485-489.

[16] V. Gregori, S. Romaguera, On completion of fuzzy metric spaces, Fuzzy Sets and Systems 130 (2002) 399-404.

[17] V. Gregori, S. Romaguera, Characterizing completable fuzzy metric spaces, Fuzzy Sets and Systems 144 (2004) 411-420.

[18] V. Gregori, S. Romaguera, Fuzzy quasi-metric spaces, Appl. Gen. Topology 5 (2004) 129-136.

[19] V. Gregori, S. Romaguera and A. Sapena, A characterization of bicompletable fuzzy quasi-metric spaces, Fuzzy Sets and Systems 152 (2005) 395-402.

[20] J. C. Kelly, Bitopological spaces, Proc. London Math. Soc. 13 (1963) 71-89.

[21] I. Kramosil and J. Michalek, Fuzzy metric and statistical metric spaces, Kybernetica 11 (1975) 326-334.

[22] H.-P. A. Künzi and C. M. Kivuvu, A double completion for an arbitrary T 0 -quasi-metric space, J. Log. Algebr. Program. 76 (2008) 251-269.

[23] H.-P. A. Künzi and C. M. Kivuvu, The B-completion of a $T_{0}$-quasimetric space, Topology Appl. 156 (2009) 2070-2081. 
[24] K. Menger, Statistical metrics, Proc. Nat. Acad. Sci. USA 28 (1942) 535-537.

[25] D. Mihet, A Banach contraction theorem in fuzzy metric spaces, Fuzzy Sets and Systems 144 (2004) 8431-439.

[26] D. Mihet, Fuzzy $\phi$-contractive mappings in non-Archimedean fuzzy metric spaces, Fuzzy Sets and Systems 159 (2008) 739-744.

[27] S. Morillas, V. Gregori and A. Hervás, Fuzzy peer groups for reducing mixed gaussian-impulsive noise from color images, IEEE transactions on Image Processing, Vol. 18, n. 7, July 2009.

[28] J. H. Park, Intuitionistic fuzzy metric spaces, Chaos, Solitons \& Fractals 22 (2004) 1039-1046.

[29] I. L. Reilly, P. V. Subrahmanyam, M. K. Vamanamurthy, Cauchy sequences in quasi-pseudo-metric spaces, Mh. Math. 93 (1982) 127-140.

[30] J. Rodríguez-López and S. Romaguera, The Hausdorff fuzzy metric on compact sets, Fuzzy Sets and Systems 147 (2004) 273-283.

[31] S. Romaguera, Left K-completeness in quasi-metric spaces, Mathematische Nachrichten 157 (1) (1992) 15-23.

[32] S. Romaguera and M. Sanchis, On fuzzy metric groups, Fuzzy Sets and Systems 124 (2001) 109-115.

[33] S. Salbany, Bitopological spaces, compactifications and completions, Mathematical Monographs Univ. Cape Town (1974).

[34] A. Sapena, A contribution to the study of fuzzy metric spaces, Appl. Gen. Topology 2 (2001) 63-76.

[35] B. Schweizer, A. Sklar, Statistical metric spaces, Pacific J. Math. 10 (1960) 314-334.

[36] R. A. Stoltenberg, Some properties of quasi-uniform spaces, Proc. London Math. Soc. 17 (1967) 226-240. 